\title{
Percepção da família quanto à doença e ao cuidado fisioterapêutico de pessoas com paralisia cerebral
}

\author{
Family perception on the disease and physiotherapeutic care of individuals with cerebral palsy \\ Percepción de la familia en cuanto a la enfermedad y al cuidado fisioterapéutico de \\ personas con parálisis cerebral
}

\author{
Raquel Mello', Sueli Mutsumi Tsukuda Ichisato", Sonia Silva Marcon"'I \\ ' Universidade Estadual do Centro-Oeste do Paraná. Guarapuava-PR, Brasil. \\ "Universidade Estadual de Maringá, Departamento de Enfermagem. Maringá-PR, Brasil. \\ "I' Universidade Estadual de Maringá, Programa de Pós-Graduação em Enfermagem, \\ Núcleo de Estudos, Pesquisa, Assistência e Apoio à Família. Maringá-PR, Brasil.
}

Submissão: 21-07-2010 Aprovação: 14-02-2012

\begin{abstract}
RESUMO
O estudo objetivou conhecer a percepção da família quanto à doença e ao cuidado fisioterapêutico de pessoas com Paralisia Cerebral. Os dados foram coletados por meio de entrevista semiestruturada junto a 24 cuidadores de crianças com paralisia cerebral que freqüentam a APAE de Guarapuava-PR. Os resultados mostraram que metade das crianças era quadriplégica e afásica e a maioria apresentava dependência para atividades da vida diária. As mães / cuidadores revelaram desconhecer a patologia de base e o tratamento fisioterapêutico que era realizado com seus filhos na Instituição. Conclui-se pela necessidade de estratégias que promovam maior interação familiar-profissional de saúde, possibilitando ao cuidador participar mais do tratamento e, por conseguinte, aprender e colocar em prática atividades benéficas para a criança.

Descritores: Paralisia cerebral; Cuidado; Modalidades de fisioterapia; Família.
\end{abstract}

\section{ABSTRACT}

The study aimed to know the perception of the family on the disease and physiotherapeutic care of individuals with Cerebral Palsy. Data was collected through semi-structured interview with 24 caretakers of children with Cerebral Palsy who are assisted at the APAE of Guarapuava -PR. The results showed that half of the children were quadriplegic and aphasic and that most of them presented dependence of care on their daily life activities. The mothers / caretakers revealed do not know the base pathology, as well as the physiotherapeutic treatment that is accomplished for their children in the institution. It is concluded that there is a need of strategies to promote more interaction between family and health professionals, making possible the caretaker to better participate on the treatment, and consequently, to learn and put into practice beneficial activities for the child.

Key words: Cerebral palsy; Care; Physical therapy modalities; Family.

\section{RESUMEN}

El estudio tuvo como objetivo conocer la percepción de la familia en cuanto a la enfermedad y al cuidado fisioterapéutico de personas con Parálisis Cerebral (PC). Los datos fueron recogidos por medio de entrevista semi estructurada junto a 24 cuidadores de niños con PC que frecuentaban la APAE (Asociación de Padres y Amigos de los Excepcionales) de GuarapuavaPR. Los resultados mostraron que mitad de los niños era cuadripléjicos y afásicos y la mayoría presentaba dependencia para actividades de la vida diaria. Las madres / cuidadores revelaron desconocer la patología de base y el tratamiento fisioterápico que es realizado con sus hijos en la institución. Se concluye por la necesidad de estrategias que promuevan mayor interacción familiar-profesional de salud, posibilitando al cuidador participar más del tratamiento, y por consiguiente, aprender y colocar en práctica actividades benéficas para el niño.

Palabras clave: Parálisis cerebral; Cuidado; Modalidades de terapia física; Família. 


\section{INTRODUÇÃO}

Paralisia cerebral (PC) é definida como um grupo de desordens do movimento e da postura conseqüentes das lesões não progressivas, que ocorreram no cérebro em fase de maturação estrutural e funcional. Trata-se de uma condição de saúde que resulta em alterações da estrutura e da função do sistema neuromusculoesquelético ${ }^{(1)}$.

A paralisia cerebral, também denominada encefalopatia crônica não progressiva da infância, é conseqüência de uma lesão estática, ocorrida no período pré, peri ou pós-natal que afeta o sistema nervoso central em fase de maturação estrutural e funcional. É uma disfunção predominantemente sensoriomotora, envolvendo distúrbios no tônus muscular, postura e movimentação voluntária( ${ }^{(1)}$. Estes distúrbios se caracterizam pela falta de controle sobre os movimentos, por modificações adaptativas do comprimento muscular e em alguns casos, chegando a resultar em deformidades ósseas ${ }^{(2)}$.

Crianças com PC, muitas vezes, podem desenvolver fraqueza muscular, dificuldades no controle entre as musculaturas agonista e antagonista, restrição da amplitude de movimento e alterações de tônus e de sensibilidade, que podem limitar a participação das mesmas em diferentes ambientes, incluindo domiciliar e escolar ${ }^{(3)}$.

Existem diversos elementos que limitam a aquisição motora na criança com paralisia cerebral, todos estes podem atuar em conjunto determinando esta limitação. Incluem aqui elementos como a motivação do paciente, a influência dos familiares, a personalidade do paciente, além da área e extensão da lesão(4).

O nascimento de uma criança "anormal" é um desses acontecimentos inesperados que põe à prova cada um de nós, pais, mães, irmãos, avós, tios, amigos, professores, profissionais da saúde, pesquisadores, gestores da saúde e da educação. Convida-nos a buscar soluções e a entender que o desafio é de todos. No entanto, a família é o primeiro núcleo social a ser intensamente "perturbada" por ele, a primeira a buscar respostas nos diversos setores da sociedade e a última a lidar com as conseqüências para toda a vida ${ }^{(5)}$.

Na reabilitação das crianças com PC, devem ser englobadas as orientações familiares, as estimulações a partir de habilidades potenciais da criança, o estado afetivo-emocional da mesma, além do quadro clínico, prognóstico e diagnóstico fisioterapêutico. Quanto mais precoce for realizada a intervenção, melhor será a resposta e aquisições motoras de cada uma $^{(6)}$.

As trocas de informações resultantes da interação fisioterapeuta/família devem estar bem estabelecidas para a definição dos objetivos funcionais, possíveis na realidade motora cognitiva e social em que a criança se encontra, essas trocas contribuirão para a superação de as dificuldades vivenciadas diariamente no relacionamento dessa família com a criança $a^{(7)}$.

Cada família é uma unidade cooperativa que compartiIha expectativas e interesses complementares. Marcada por diferenças de gênero, geração, classe social, entre outras. A família se vê às voltas com o difícil desafio de socializar uma criança diferente ${ }^{(5)}$.
A criança com paralisia cerebral que realiza algum programa de tratamento, independente do profissional e da técnica utilizada, necessita que sua mãe possua esclarecimento tanto da patologia, como da terapia instituída, para que esta durante e após a sessão de tratamento tenha um papel importante, não apenas como prestadora dos cuidados básicos no dia-a-dia, mas como peça fundamental durante o processo de reabilitação( ${ }^{(8)}$.

Para atender esta demanda existe a Escola de Educação Especial Anne Sullivan, Associação de Pais e Amigos dos Excepcionais (APAE), no município de Guarapuava-PR, em que uma das autoras deste estudo foi supervisora de estágio do Curso de Fisioterapia, da Universidade Estadual do Centro Oeste. Durante a supervisão Ihe inquietava o quanto a família era importante na reabilitação dos Portadores de Necessidade Especial (PNE). Porém por que não participavam efetivamente na execução dos exercícios de fisioterapia?

Com o intuito de programar medidas de prevenção que possibilitassem melhorar a qualidade do atendimento fisioterapêutico ao PNE, especialmente, na parte motora e, consequentemente, nas atividades da vida diária desse cliente, percebeu-se a necessidade de conhecer o conhecimento do familiar (cuidador) em relação aos cuidados fisioterapêuticos realizados ao Portador de Necessidade Especial, pois os cuidadores podem ser auxiliares na reabilitação, visto que passam a maior parte do tempo com a criança, podendo assim, facilitar o tratamento.

\section{METODOLOGIA}

Trata-se de um estudo exploratório descritivo de natureza qualitativa, realizado com familiares (cuidadores) de alunos da Escola de Educação Especial Anne Sullivan, Associação de Pais e Amigos dos Excepcionais (APAE), situada no município de Guarapuava (PR). A APAE é um estabelecimento de ensino para Portadores de Necessidades Especiais, que presta serviços de reabilitação e habilitação. Possui uma equipe multiprofissional e seus recursos financeiros são provenientes do Sistema Único de Saúde (SUS), doações sociais, contribuintes e convênio com o Estado e o Município.

A clientela assistida na Instituição é acompanhada por uma equipe composta por fisioterapeutas, fonoaudiólogos, psicólogos, terapeuta ocupacional, assistente social, educador físico e por acadêmicos do último ano do curso de fisioterapia, juntamente com os professores supervisores por intermédio da Universidade Estadual do Centro Oeste.

Os familiares foram convidados a participar da pesquisa de acordo com o critério de inclusão, ou seja, que fossem responsáveis pelos alunos com PC assistidos na instituição e que tivessem assinado ou tivessem a impressão datiloscópia no Termo de Consentimento Livre e Esclarecido quando necessária.

A coleta de dados foi realizada na segunda quinzena de outubro de 2008 nas dependências da instituição, mediante uma entrevista semiestruturada (gravada) com o familiar responsável pela criança e consulta aos prontuários dos alunos. As entrevistas foram realizadas individualmente, após o 
horário das reuniões de pais que ocorrem trimestralmente, nos turnos da manhã e da tarde. Para uma melhor compreensão e tratamento dos dados, as entrevistas foram transcritas na integra e agrupadas por aproximação das respostas dadas pelos cuidadores.

O desenvolvimento do estudo ocorreu em conformidade com o preconizado pela Resolução 196/96 do Conselho Nacional de Saúde e o projeto foi submetido à apreciação e aprovado pelo Comitê Permanente de Ética em Pesquisa com Seres Humanos (COPEP) da Universidade Estadual de Maringá, (Parecer $n^{\circ}$ 551/2008). Todos os participantes assinaram o termo de consentimento livre e esclarecido em duas vias.

\section{RESULTADOS E DISCUSSÃO}

\section{Caracterização das pessoas com Paralisia Cerebral}

A idade dos Portadores de Necessidade Especial variou de sete meses a 42 anos. Quase metade (43,30\%) destes indivíduos apresentava comprometimento nos quatro membros, ou seja, são classificados como quadriplégicos. Mais da metade $(57,69 \%)$ deles apresentava as deficiências somente motoras, para os demais, além desta deficiência também apresentam alterações mentais $(19,23 \%)$, visuais $(11,53 \%)$ e visuais e mentais conjuntamente $(11,53 \%)$. Estes resultados são coerentes com o estudo encontrado onde a quadriplegia ocorre de nove a $43 \%$ dos pacientes onde as lesões são difusas no sistema piramidal com intensas retrações em semiflexão, disfagia e disartria, podendo ocorrer ainda microcefalia e deficiência mental ${ }^{(9)}$.

Das pessoas com paralisia cerebral quinze tinham afasia e sete disartria, demonstrando assim, um grande déficit de linguagem nesses pesquisados. A afasia pode ser definida como a perda ou redução da capacidade de processar linguagem como resultado de uma lesão cerebral, já a disartria, como um grupo de perturbações da fala que resulta da falha no controle motor (fraqueza, lentidão ou incoordenação) do mecanismo da fala, devido à lesão central e/ou periférica do sistema nervoso ${ }^{(10)}$.

\section{Caracterização do cuidador e da família}

Foram entrevistados 24 cuidadores, visto que duas famílias possuíam duas crianças com paralisia cerebral. A idade dos cuidadores variou de 20 a 79 anos (média de 41,12 anos). A grande maioria $(70,83 \%$ ) tinha baixa escolaridade (fundamental incompleto) e eram designadas como do lar. Dezesseis cuidadores eram casados e o grau de parentesco com o indivíduo com PC mais freqüente era de mãe $(83,33 \%)$, o que pode levar a inferir que a maioria das mães não trabalhava fora e ficavam cuidando das crianças que tinham necessidades especiais. Isso já foi observado no estudo realizado com mães de PC sobre o impacto da dinâmica familiar e o conhecimento da patologia, onde o impacto da aceitação e compreensão das informações quanto ao filho com PC é tão grande que se percebe alterações na rotina diária, nos sonhos e projetos de cada membro da família, que se desestrutura sendo necessário longo processo para que retome o equilíbrio(11).

A família desempenha um papel importante na provisão de cuidado informal para seus membros. Há um reconhecimento geral, hoje em dia, de que ela está no centro das funções do cuidado. Uma grande parte do cuidado acontece no lar. A vida quotidiana doméstica é caracterizada pelo atendimento às necessidades físicas e psicológicas dos diferentes membros da família. É no seu contexto social que se salvaguarda a saúde e se lida com as doenças ${ }^{(12)}$.

Essas famílias têm como dependentes até três menores $(70,83 \%)$, sendo que dezenove entrevistados têm como renda familiar de até dois salários mínimos, sendo um deles o salário do filho com necessidades especiais, e o outro possivelmente do pai ou responsável. O mesmo foi encontrado em um estudo sobre as atividades e participação de crianças com PC, onde a maioria das famílias arrecada mensalmente em média dois salários mínimos ${ }^{(13)}$.

Essas famílias têm como dependentes até três menores $(70,83 \%)$, sendo que dezenove entrevistados têm renda familiar de até dois salários mínimos, sendo um deles o salário do filho com necessidades especiais, e o outro possivelmente do pai ou responsável. $\mathrm{O}$ mesmo foi encontrado em um estudo sobre as atividades e participação de crianças com PC, onde a maioria das famílias arrecada mensalmente em média dois salários mínimos ${ }^{(13)}$.

Das famílias pesquisadas, grande parte possui casa própria (75\%), sendo que doze casas têm entre cinco e seis peças, 23 têm banheiro com água encanada e para 16 famílias o acesso da casa é asfaltado e possui sistema de esgoto na rua. Como visto em outro estudo, o ambiente serve como determinante da saúde, a habitação se constitui em um espaço de construção da saúde e consolidação do seu desenvolvimento. A família tem seu assento na habitação e, com isto, a habitação é o espaço essencial, o veículo da construção e desenvolvimento da saúde da família ${ }^{(14)}$.

Em relação à adaptação da casa e cômodos para realizar as transferências das pessoas com paralisia cerebral, 22 responderam que a casa não está adaptada, isso pode ser um fator perturbador para o familiar, pois nas atividades da vida diária a maioria dessas pessoas é totalmente dependente. Em crianças com PC, o ambiente domiciliar deve ser consistentemente avaliado, de forma a indicar as modificações necessárias que visem à promoção da participação e independência dessas crianças em suas atividades cotidianas ${ }^{(15)}$.

\section{Dependência para atividades da Vida Diária das pessoas com Paralisia Cerebral}

Ao serem questionados em relação às atividades da vida diária (Tabela 1), os cuidadores revelaram que a maioria das pessoas com PC era dependente de cuidados, pois, não tomava banho sozinho $(75 \%)$, não se vestia sozinho (75\%), usava fraldas $(62,50 \%)$, não se alimentava sozinho (58,33\%), não passava sozinho da cadeira de rodas para a cama $(41,66 \%)$. Estes resultados são concordantes com os encontrados em estudo que comparou o desempenho funcional de crianças normais e crianças com PC. De acordo com o estudo, em crianças com PC o desempenho dessas atividades é, muitas vezes, a principal queixa de pais e familiares, e/ou da própria criança. Elas apresentam um repertório de habilidades funcionais de vida diária diferenciado, e tem dificuldade relativa para desempenhar diversas atividades de autocuidado ${ }^{(2)}$. 
Tabela 1 - Distribuição das Atividades da Vida Diária que a pessoa com PC realiza. Guarapuava (PR), 2008.

\begin{tabular}{|c|c|c|}
\hline & $N^{0}$. de respostas & $\%$ \\
\hline \multicolumn{3}{|l|}{ Alimentação } \\
\hline Alimenta-se sozinho & 8 & 33,3 \\
\hline Não consegue ajudar na alimentação & 16 & 66,7 \\
\hline \multicolumn{3}{|l|}{ Banho } \\
\hline Toma banho sozinho & 2 & 8,3 \\
\hline Não consegue ajudar no banho & 22 & 91,7 \\
\hline \multicolumn{3}{|l|}{ Vestir-se } \\
\hline Veste-se sozinho & 1 & 4,1 \\
\hline Não consegue ajudar & 23 & 95,9 \\
\hline \multicolumn{3}{|l|}{ Uso de fraldas } \\
\hline Usa fraldas & 15 & 62,5 \\
\hline Não usa fraldas & 9 & 37,5 \\
\hline \multicolumn{3}{|l|}{ Uso de vaso sanitário } \\
\hline Faz uso do vaso sanitário sozinho & 6 & 25,0 \\
\hline Não faz uso do vaso sanitário sozinho & 18 & 75,0 \\
\hline \multicolumn{3}{|l|}{ Transferências } \\
\hline Passa sozinho da cadeira de rodas para a cama & 2 & 8,3 \\
\hline Não passa sozinho da cadeira de rodas para a cama & 10 & 41,7 \\
\hline Não utiliza cadeira de rodas & 12 & 50,0 \\
\hline \multicolumn{3}{|l|}{ Deambulação } \\
\hline Caminha sozinho & 3 & 12,5 \\
\hline Não consegue caminhar & 15 & 62,5 \\
\hline Caminha com auxílio & 6 & 25,0 \\
\hline
\end{tabular}

hidrocefalia; três cuidadores responderam que não sabiam qual era a doença e os outros quatro cuidadores cada um verbalizou uma patologia diferente, tais como: "encefalia", epilepsia associada à rubéola, "ataque" e atrofia cerebral.

Em relação às respostas apresentadas observou-se inicialmente que as mães apresentavam certa confusão entre diagnóstico e etiologia da doença. Algumas não tinham noção do diagnóstico e outras realmente não soberam responder. Contudo, é interessante salientar que não foi observada diferença nas características das mães que souberam informar corretamente o diagnóstico das que não souberam, visto que entre as que souberam informar corretamente só uma estava cursando ensino superior, outra o ensino médio e todas as demais tinham no máximo ensino fundamental incompleto, duas inclusive eram analfabetas e todas eram do lar. Entre as três que não souberam, duas eram analfabetas e uma tinha ensino fundamental incompleto.

Estes resultados reforçam a necessidade, de salientar a necessidade de as informações referentes ao problema de saúde da criança com PC sejam dadas por profissionais experientes, em linguagem simples e acessível, para pos-

De fato, a criança com PC, em decorrência das limitações, torna-se cada vez mais dependente de sua mãe e de seus familiares, o que pode dificultar o processo de individualização e da forma que está sendo abordada à questão da independência nas suas atividades básicas da vida diária ${ }^{(16)}$. Neste contexto é comum as mães que relataram alteração no desenvolvimento dos menores, quando comparados com outras crianças de idade cronológica semelhante, e apesar disto, a maioria delas demonstra despreparo nas atividades como as de assistência na higiene, alimentação, vestuário, transferências e atividades lúdicas ${ }^{(8)}$.

As atividades de vida diária (AVD) que fazem parte do cotidiano infantil incluem tarefas de auto-manutenção como banho, vestuário, alimentação, uso do banheiro, higiene oral e comunicação. O desempenho de tais atividades é importante para que a criança seja capaz de satisfazer suas necessidades básicas, garantindo-lhe maior independência e participação em seu ambiente domiciliar ${ }^{(17)}$.

\section{Percepção do cuidador (familiar)}

Ao investigar junto aos cuidadores o conhecimento que eles tinham sobre a doença, constatou-se que apenas nove deles identificam o problema de saúde atual como PC, oito cuidadores fizeram referência à etiologia uma vez que afirmaram que a criança teve meningite, problemas no parto, hipoplasia de corpo caloso, hipóxia cerebral do lado esquerdo e sibilitar a compreensão adequada do que está sendo falado (12). Além disso, entende-se que estas informações devam ser repetidas de tempos em tempos visto que existe um despreparo emocional e/ou técnico de profissionais de saúde para detectar, em comunicar e em orientar os pais quanto à paralisia cerebral, existindo também uma "barreira lingüística" que separa os profissionais da saúde do doente em especial àqueles de classes populares ${ }^{(18)}$.

Para nove responsáveis pela criança com PC a doença [...] não tem cura [...], já sete responsáveis acreditam que [...] tem cura sim [...], e três deles apresentam respostas do tipo [...] não sei se a doença tem cura [...]. Essas respostas nos levam a refletir sobre a dificuldade que os cuidadores apresentam em relação ao entendimento sobre a doença e seu prognóstico, questões estas que já haviam sido evidenciadas em estudo que avaliou o nível de conscientização de mães de crianças com PC espástica ${ }^{(8)}$.

Essa falta de entendimento fica ainda mais evidente diante das respostas apresentadas ao questionamento sobre o que poderia ser feito pela criança com necessidades especiais, visto que oito deles responderam não saber, cinco referiram que a criança deveria fazer fisioterapia, três cuidadores referiram que eles deveriam estimular essas crianças.

Expectativas positivas ou negativas quanto ao desenvolvimento e o futuro do filho podem ser influenciadas pelo entendimento das informações passadas, prejudicando em algum momento, o oferecimento dos recursos especializados 
necessários ao bom desenvolvimento dessa criança. O tratamento da PC deve ser realizado por uma equipe multiprofissional e o mais precocemente possível e tem por objetivo um melhor desenvolvimento neuropsicomotor, promovendo o melhor nível de independência possível para essas crianças, que devem ser contínuas para manter todas as conquistas alcançadas com essas crianças ${ }^{(8,12)}$.

Em estudo sobre os significados e crenças do cuidador familiar, foi evidenciado que há necessidade de acompanhamento pelas equipes de saúde aos cuidadores, pois a insegurança de vivenciar sentimentos novos e dolorosos envolvidos no cuidado exige um preparo emocional dos cuidadores que nem sempre recebem essa atenção da equipe ${ }^{(19)}$.

Quando questionados quanto ao conhecimento do que o fisioterapeuta faz na APAE, quatorze apresentaram respostas do tipo "faz exercícios e ajuda a desenvolver a criança". Os demais referiram não ter conhecimento daquilo que é realizado numa sessão de fisioterapia, sendo que seis referiram nunca ter participado/assistido um atendimento fisioterapêutico. A maioria das pessoas participantes do estudo possuía baixa condição socioeconômica, o que justifica o fato de as crianças com PC não freqüentarem outros serviços de fisioterapia que não o da APAE e, por conseguinte, desconhecerem o que é realizado em uma sessão de fisioterapia.

Talvez a falta de conhecimento relatado também se deva à elevada demanda de pacientes e a insuficiência de espaço físico, o qual não permite a entrada de acompanhantes nas sessões de fisioterapia. Embora a maioria dos alunos faça o deslocamento de casa até a escola em ônibus especial, constatamos que até mesmo as poucas mães que levavam seus filhos até a instituição e lá permaneciam até o término das atividades não podiam acompanhar o atendimento fisioterapêutico. Este fato causa um distanciamento na interação fisioterapeuta-cuidador, pois os pais poderiam fornecer informações importantes ao fisioterapeuta, afinal é ele quem convive no dia-dia com a criança. Por outro lado o fisioterapeuta poderia orientar a realização de exercícios e posicionamentos adequados para serem utilizados no domicílio. Cabe salientar que o tratamento da PC deve ser recomendado o mais precocemente possível e por uma equipe multiprofissional, conforme já mencionado, também deve enfocar a relação mãe-filho como estratégia de maneira completa nestes $\operatorname{casos}^{(8,20)}$.

Os cuidadores foram questionados quanto à possibilidade de repetir em casa os exercícios repassados pelo fisioterapeuta. Todos os entrevistados responderam que poderiam estar tentando aplicar esses exercícios no domicílio. Na verdade os cuidadores até tinham a intenção de participar da terapia, mas não haviam sido convidados, e para o fisioterapeuta a ausência do cuidador na sessão denotava falta de interesse o que na verdade, era a falta de espaço na clínica que impossibilitava esse intercambio de informações. Em estudo realizado com as mães de crianças com PC, foi encontrado que todas as mães explanaram a importância de sua participação no tratamento do seu filho, quer seja nos cuidados diários, ou realizando práticas orientadas pelos profissionais ${ }^{(8)}$.

Quando questionados sobre como poderiam melhorar a qualidade de vida das crianças, dez cuidadores responderam não saber; outros fizeram referência a uma educação junto à criança:

[...] auxiliando e levando nos atendimentos [...] (cinco cuidadores);

[...] estimulando em casa e brincando com eles [...] (dois cuidadores);

[...] incentivando e ajudando [...] (dois cuidadores);

Alguns percebiam a necessidade de existir algumas mudanças pessoais para então ajudar a criança:

[...] aprender a fazer os exercícios [...] (um cuidador);

[...] tirando carteira de motorista para conduzir meu filho

[...] (um cuidador);

[...] deixei o trabalho para cuidar dele [...] (um cuidador).

As respostas desses familiares denotam o cuidado e a intenção da maioria desses pais em melhorar a qualidade de vida das crianças com PC. Em estudo realizado sobre participação da família no trabalho fisioterapêutico em crianças com PC, a maioria das mães afirmou perceber resultados positivos com o tratamento de fisioterapia e que a partir do início das atividades fisioterapêuticas, os filhos apresentaram melhoras mediante alguns desempenhos que não apresentavam anteriormente, como sentar, rolar, ficar em pé, engatinhar, andar, andar com apoio e pegar objetos ${ }^{(7)}$.

A atuação dos pais no tratamento pode atingir mais do que o previsto, principalmente quando se trata de uma criança motivada, que convive com uma família que Ihe auxilia, ama e incentiva. Mesmo diante das incapacidades, deve-se estimular e incentivar a família para a realização das atividades no domicílio pois o carinho e atenção da família são importantes para reforçar o vínculo com a criança, o que por sua vez favorece o seu desenvolvimento global $^{(7,8)}$.

\section{CONSIDERAÇÕES FINAIS}

A criança com paralisia cerebral tem direito à avaliação e a cuidados durante toda sua vida. O tratamento constante e os programas de apoio são essenciais para minimizar a deterioração e o efeito negativo que isso tem sobre a qualidade de vida, assim como sobre recursos financeiros e outros.

Nesta pesquisa, verificou-se que a percepção da família e do cuidado fisioterapêutico ainda está abaixo do esperado, pois a grande maioria dos cuidadores não apresenta o grau necessário de informações sobre a patologia e o tratamento fisioterapêutico. Isso revela a importância desses cuidadores em obter conhecimento sobre a PC, pois podem assumir um papel mais ativo na reabilitação.

Os dados revelaram a falta de interação entre o cuidador e o fisioterapeuta, provavelmente em decorrência da falta de espaço físico, possibilitando àquelas mães que permaneciam na escola a participar das sessões de fisioterapia e também devido a elevada demanda que não permitia tempo suficiente para os fisioterapeutas estabelecerem interações com as famílias. Assim, sugerem-se estratégias que promovam mais interação familiar-fisioterapeuta, possibilitando ao cuidador participar do tratamento, aprender e colocar 
em prática atividades benéficas para a criança. Além disso, o estudo evidenciou a importância de conhecer, orientar e ouvir o cuidador, pois ele é a pessoa que está em contato mais próximo com a criança que tem paralisia cerebral e pode ser o maior aliado na melhoria da qualidade de vida desta criança.

\section{REFERÊNCIAS}

1. Bax M, Goldstein M, Rosenbaum P, Leviton A, Paneth N, Dan B, et al. Proposed definition and classification of cerebral palsy. Dev Med Child Neurol 2005;47:571-6.

2. Mancini M, Fiúza P, Rebelo J, Magalhães L, Coelho Z, Paixão M. Comparação do Desempenho de Atividades Funcionais Em Crianças Com Desenvolvimento Normal e Crianças Com Paralisia Cerebral. Arq. Neuro-Psiquiatr 2002;60.

3. Beckung E, Hagberg G. Neuroimpairments, activity limitations, and participation restrictions in children with cerebral palsy. Dev Med Child Neurol. 2002;44:309-16.

4. Bartlett D, Palisano R. Physical Therapists' Perceptions of Factors Influencing the Acquisitions of motor abilities of children of cerebral palsy: Implications for clinical reasoning. Phys Ther 2002;82:237-47.

5. Cavalcante F. Família, subjetividade e linguagem: gramáticas da criança "anormal". Ciênc Saúde Coletiva 2001;6(1):125-37.

6. Bartlett D, Palisano R. A Multivariate model of determinants of motor change for children with cerebral palsy. Phys Ther 2000;80:598-611.

7. Sari FL, Marcon SS. Participação da família no trabalho fisioterapêutico em crianças com paralisia cerebral. Rev Bras Crescimento Desenvolv Hum 2008;18(3):229-39.

8. Gração DC, Santos MGM. A percepção materna sobre a paralisia cerebral no cenário da orientação familiar. Fisioter Mov 2008;21(2):107-13.

9. Leite JMRS, Prado GF. Paralisia cerebral: aspectos fisioterapêuticos e clínicos. Arq Neurociênci 2005;41.

10. Leal G, Martins IP. Avaliação da afasia pelo Médico de Família. Rev Port Clin Geral 2005;21:359-64.

11. Petean EBL, Murata MF. Paralisia Cerebral: conhecimento das mães sobre o diagnóstico e o impacto deste na dinâmica familiar. Paidéia (Ribeirão Preto) 2000;10(19):40-6
12. Serapioni M. O papel da família e das redes primárias na reestruturação das políticas sociais. Ciênc Saúde Coletiva 2005; 10(4):243-53.

13. Brasileiro IC, Moreira TMM, Jorge MSB, Queiroz MVO, Mont'Alverne DGB. Atividades e participação de crianças com Paralisia Cerebral conforme a Classificação Internacional de Funcionalidade, Incapacidade e Saúde. Rev Bras Enferm 2009;62(4):503-11.

14. Cohen S, Cynamon S E, Kligerman D C, Assumpção R F. Habitação saudável no Programa Saúde da Família (PSF): uma estratégia para as políticas públicas de saúde e ambiente. Ciênc Saúde Coletiva 2004; 9(3):807-13.

15. Guerzoni VPD, Barbosa AP, Borges ACC, Chagas PSC, Gontijo APB, Eterovick F, et al. Análise das intervenções de terapia ocupacional no desempenho das atividades de vida diária em crianças com paralisia cerebral: uma revisão sistemática da literatura. Rev Bras Saúde Matern Infant 2008;8(1):17-25.

16. Ones K, Yilmaz E, Cetinkaya B, Caglar N. Assessment of the quality of life of mothers of children with cerebral palsy (primary caregivers). Neurorehabil Neural Repair 2005;19(3):232-7.

17. Spackman CS, Maureen EC, Crepeau EB. Willard \& Spackman. Terapia ocupacional. Rio de Janeiro: Guanabara Koogan; 2002.

18. Dagenais L, Hall N, Majnemer A, Birnbaum R, Dumas $\mathrm{F}$, Gosselin J, et al. Communicating a diagnosis of cerebral palsy: caregiver satisfaction and stress. Pediatr Neurol 2006;35(6):408-14.

19. Machado ALG, Freitas CHA, Jorge MSB. O fazer do cuidador familiar: significados e crenças. Rev Bras Enferm 2007;60(5):530-4.

20. Powell L, Barlow J, Cheshire. The training and support programme for parents of children with cerebral palsy: aprocess evaluation. Compl Ther Clin Pract 2006;(12)192-9. 